\title{
On a weaker notion of ring stability for mixed traffic with human-driven and autonomous vehicles
}

\author{
Vittorio Giammarino*, Maolong Lv† Simone Baldi; \\ Paolo Frasca ${ }^{\S}$ and Maria Laura Delle Monacheף
}

\begin{abstract}
This article considers criteria to determine when stop-and-go waves form in platoons of human-driven vehicles, and when they can be dissipated by the presence of an autonomous vehicle. Our analysis takes the start from the observation that the standard notion of string/ring stability definition, which requires uniformity with respect to the number of vehicles in the platoon, is too demanding for a mixed traffic scenario. The setting under consideration is the following: the vehicles run along a ring road and the human-driven vehicles obey a combined follow-the-leader and optimal velocity model, while the autonomous vehicle obeys an appropriately designed model. The criteria are tested on a linearized version of the resulting platoon dynamics and simulation tests using nonlinear model are carried out.
\end{abstract}

\section{Introduction}

Currently, the transportation system is undergoing a major transition from full human controlled vehicles to automated vehicles (AV) or automated infrastructure, some examples being adaptive cruise control [1, 2], cooperative adaptive cruise control (CACC) [3, 4], ramp meters or variable speed limit systems [5, 6, 7]. As automation increases, new opportunities are arising to use AV to control the traffic flow. Interestingly, field experiments with commercial adaptive cruise control vehicles illustrate how current technology may amplify traffic oscillations (stop-and-go traffic waves), while correctly designed CACC systems can dissipate them [8, 9]. Considering this likely new direction of traffic control, the main contribution of this article is to propose a new approach to analyze traffic oscillation and to assess AV control algorithms designed to dissipate stop-and-go traffic waves. In the seminal experiments of Sugiyama et al. [10], conducted on a single lane circular track with real human drivers, human driving behavior alone is shown to be sufficient to trigger stop-and-go waves that travels against the traffic flow. Subsequent to [10], in [11] a human vehicle is replaced by an AV, showing how a proper controller is able to dissipate the occurring waves.

Motivated by these experiments, we analyse a linearized version of the control algorithms developed in [12]. First we give a new definition of string stability on the ring (ring stability) motivated by the fact that uniformity in the number of vehicles in the platoon, as required in standard notions, is not appropriate for mixed traffic scenarios (human/automated vehicles). Afterwards, we model the vehicular traffic at the microscopic scale using the combination of two well-known microscopic models, namely i) the Bando or optimal velocity (OV) model [13] and the ii) follow the leader (FTL) [14]. With the human model defined, we show that its linearization at the equilibrium can be used to investigate stability in the platoon of vehicles on the ring. This result gives indications on how designing an AV controller to dissipate the stop and go traffic waves created in the ring.

\section{Stability notions for interconnected systems}

In this section, the standard definitions for stability for systems interconnected on a string and on a ring topology are recalled. Regardless of the fact that the platoon is placed on a string or on a ring, Fig. 1 depicts the interaction between two adjacent vehicles, where $y_{i}$ and $u_{r}$ are respectively the output of the $i$-th vehicle

\footnotetext{
${ }^{*} \mathrm{~V}$. Giammarino is with the Division of Systems Engineering, Boston University, 15 St Marys St, Brookline, MA 02446, USA and was with Delft Center for Systems and Control, Delft University of Technology (TU Delft), 2628 CD Delft, The Netherlands vittoriogiammarino@gmail.com

$\dagger^{\dagger}$ M. Lv is with Delft Center for Systems and Control, Delft University of Technology (TU Delft), 2628 CD Delft, The Netherlands M.Lyu@tudelft.nl

$\ddagger_{S}$. Baldi (corresponding author) is with School of Mathematics, Southeast University, Nanjing, China, and with Delft Center for Systems and Control, Delft University of Technology (TU Delft), 2628 CD Delft, The Netherlands s.baldi@tudelft.nl

$\S$ P. Frasca is with Univ. Grenoble Alpes, CNRS, Inria, Grenoble INP, GIPSA-Lab, 38000 Grenoble, France paolo.frasca@gipsa-lab.fr

ॠM. L. Delle Monache is with Univ. Grenoble Alpes, Inria, CNRS, Grenoble INP, GIPSA-Lab, 38000 Grenoble, France ml.dellemonache@inria.fr
} 


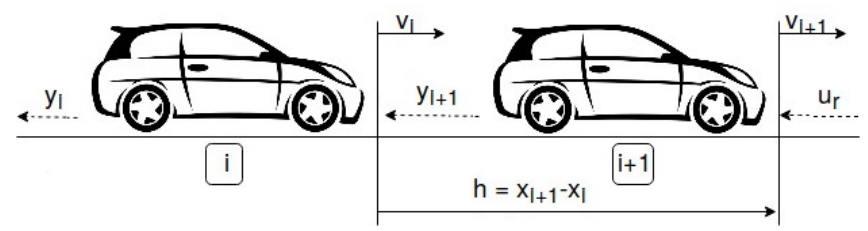

Figure 1: Platoon of Vehicles

and an exogenous input acting on the $i+1$-th vehicle. Typical outputs and inputs are velocities and acceleration commands, respectively, although different choices are also possible [15]: for example, $u_{r}(s)$ can also be seen as an exogenous disturbance acting on the $i+1$-th vehicle. To study stability of interconnected system it is crucial to define

$$
y_{i}(t)=\Gamma_{i}(s) y_{i+1}(t),
$$

which represents how the output of each vehicle affects the following vehicle. Note that we will be using a hybrid notation where the Laplace operator $s$ (frequency domain) appears together with the time domain. This typically helps to streamline the notation.

\subsection{Uniform string stability}

When an exogenous input $u_{r}$ acts on a platoon of vehicles on a string, a string stability definition can be formulated with respect to the input (cf. [15]). A platoon on a string can be represented as the following cascade state-space system:

$$
\begin{aligned}
\dot{\chi}_{N} & =f_{r}\left(\chi_{N}, u_{r}\right) \\
\dot{\chi}_{i} & =f_{i}\left(\chi_{i+1}, \chi_{i}\right), \quad y_{i}=h\left(\chi_{i}\right) .
\end{aligned}
$$

Here, $N \in \mathbb{N}$ is the number of vehicles in the platoon, $u_{r} \in \mathbb{R}^{q}$ is the external input, $\chi_{i} \in \mathbb{R}^{n}, i \in\{1, \ldots, N\}$, is the state vector (typically position and velocity of each vehicle), and $y_{i} \in \mathbb{R}^{l}, i \in\{1, \ldots, N\}$, is the output. Moreover, $f_{r}: \mathbb{R}^{n} \times \mathbb{R}^{q} \mapsto \mathbb{R}^{n}, f_{i}: \mathbb{R}^{n} \times \mathbb{R}^{n} \mapsto \mathbb{R}^{n}, i \in\{1, \ldots, N-1\}$, and $h: \mathbb{R}^{n} \mapsto \mathbb{R}^{l}$. The functions $f_{r}, f_{i}$ and $h$ describe the dynamics of a car-following model $[15,16]$. Linearizing the interconnected car-following model (2) at its equilibrium leads to the following transfer function:

$$
y_{N}(t)=P_{N}(s) u_{r}(t), \quad y_{i}(t)=P_{N}(s) \prod_{j=i}^{N-1} \Gamma_{j}(s) u_{r}(t)
$$

At this point, a definition of uniform string stability as given in [15] can be provided.

Definition 1 (Uniform String Stability). Let (3) represent a linear one-vehicle look-ahead interconnected system whose input-output relation is described by (3). Assume $P_{i}(s)$ invertible, for all $i \in\{1, \ldots, N\}$. Then the system (3) is said to be uniformly string stable if

$$
\begin{aligned}
& \left\|P_{N}(j \omega)\right\|_{\infty} \quad \text { is finite } \forall N \in \mathbb{N} ; \\
& \left\|\Gamma_{i}(j \omega)\right\|_{\infty} \leq 1 \quad \forall i \in\{1, \ldots, N-1\}, \quad \forall N
\end{aligned}
$$

The definition of uniform string stability is also known as $L_{2}$ string stability, motivated by the requirement of energy dissipation of a disturbance throughout the string.

\subsection{Uniform ring stability}

Analogously, when an exogenous input $u_{r}$ acts on a platoon of vehicles on a ring road, a ring stability definition can be formulated with respect to the input (cf. [17]). A platoon on a ring road can be represented as the following cascade state-space system:

$$
\begin{aligned}
\dot{\chi}_{N} & =f_{r}\left(\chi_{N}, \chi_{1}, u_{r}\right), \\
\dot{\chi}_{i} & =f_{i}\left(\chi_{i+1}, \chi_{i}\right), \quad y_{i}=h\left(\chi_{i}\right),
\end{aligned}
$$

where (6) is analogous to (2) with the difference that, in view of the ring topology, the dynamics of vehicle $N$ depend both on $u_{r}$ and on the dynamics of vehicle 1 . With $N$ vehicles running along a ring, we have $N+i=i$.

Differently from (3), after linearizing the system at its equilibrium, the transfer function from $u_{r}$ to $y_{i}$ is:

$$
y_{i}(s)=F_{i}^{(N)}(s) u_{r}(s)
$$




$$
F_{i}^{(N)}(s)=\frac{\prod_{j=i}^{N-1} \Gamma_{j}(s)}{1-\prod_{j=1}^{N} \Gamma_{j}(s)} P_{N}(s)
$$

Note that $\prod \emptyset=1$. We are going to give a definition for uniform string stability on the ring, which, for the sake of brevity is named uniform ring stability. This definition is inspired by the results obtained in [18] for a homogeneous platoon $\left(\Gamma_{i}=\Gamma, \forall i\right)$, for which $F_{i}^{(N)}(s)$ is

$$
F_{i}^{(N)}(s)=\frac{\Gamma^{N-i}(s)}{1-\Gamma^{N}(s)} P_{N}(s)
$$

Definition 2 (Uniform Ring Stability). Consider a homogeneous platoon $F_{i}^{(N)}(s)$ defined in (9) for all $i, N$ with $i \leq N$ and assume $\|\Gamma\|_{\infty} \leq 1$. Then, the platoon is said to be uniformly ring stable if there exists $c>0$ such that $\left\|F_{i}^{(N)}(s)\right\|_{\infty} \leq c$ for all $i, N, i \leq N$.

Definition 2 is based on the analysis in [18] which leads to implications that are worth to be highlighted. First of all, assuming uniform string stability in the platoon, i.e. $\|\Gamma\|_{\infty} \leq 1$, implies that all $F_{i}^{(N)}(s)$ have their poles in the open-left-half plane. We find the scope of application of Definition 2 too narrow for our goals. Firstly, Definition 2 does not consider heterogeneous platoons, such as mixed platoons with human and autonomous vehicles, as we intend to do it. Secondly, Definition 2 does not take into account those cases where, although $\|\Gamma\|_{\infty}>1$ and therefore a uniform-over- $N$ bound does not exist, disturbances are not amplified throughout a platoon of a given size. This latter case is specially relevant for mixed platoons, since human vehicles are naturally prone to exhibit $\left\|\Gamma_{i}\right\|_{\infty}>1$.

\subsection{Weak ring stability}

In view of the limitations that we discussed above, it becomes relevant to look for a weaker version of ring stability, where we essentially renounce to uniformity of bounds with respect to $N$, thereby addressing all the cases not considered in Definition 2.

Definition 3 (Weak Ring Stability). Fix $N$ and consider $F_{i}^{(N)}(s)$ defined in (8) for all $i$ with $i \leq N$. Assume that $F_{i}^{(N)}(s)$ has all its poles in the open-left-half plane. Then, the platoon is said to be weakly ring stable if $\left\|F_{i}^{(N)}\right\|_{\infty} \leq\left\|F_{i+1}^{(N)}\right\|_{\infty}$ for all $i$, with $i \leq N$.

Remark 1 (Weak string stability counterpart). The analogous of Definition 3 can be formulated also for the string case. This would amount to checking the amplification of $\left\|P_{N} \prod_{j=i}^{N-1} \Gamma_{j}\right\|_{\infty}$ throughout the platoon. Clearly, such a definition would allow some $\Gamma_{i}$ to have $H_{\infty}$ norm greater than 1 , provided that this does not result in an overall amplification of the peak.

Notably, as compared to Definition 2, we have that Definition 3: can address heterogeneous platoons, covers those cases where the equilibrium is Lyapunov stable, although $\left\|\Gamma_{i}\right\|_{\infty}>1$, covers those cases where the effect of the disturbance is not amplified for platoons of fixed size, although a uniform bound over $N$ does not exist.

In the following two sections, we will see that all these scenarios can actually occur in mixed platoon conditions with human and autonomous vehicles.

\section{Human platoon}

The model of a human-driven vehicle is taken to be a FTL-OV car following model as in [12].

$$
\begin{aligned}
& \dot{x}_{i}=v_{i} \quad i \in\{1, \ldots, N\} \\
& \dot{v}_{i}=a \frac{v_{i+1}-v_{i}}{\left(x_{i+1}-x_{i}\right)^{2}}+b\left[V\left(x_{i+1}-x_{i}\right)-v_{i}\right]
\end{aligned}
$$

where $V\left(x_{i+1}-x_{i}\right)$ is a non-linear function determining the desired speed

$$
V(h)=v_{\max } \frac{\tanh \left(h-l_{v}-d_{s}\right)+\tanh \left(l_{v}+d_{s}\right)}{1+\tanh \left(l_{v}+d_{s}\right)}
$$

where $l_{v}$ is the vehicle length and $d_{s}>0$ is a safety distance between cars. Note that (11) tends to zero for small headways and approaches $v_{\max }$ for large headways.

The variables $a$ and $b$ in (10) represent peculiarities of human driving style and will be object of further investigation. To the purpose of analysis, let us derive the linearization of (10)

$$
\begin{aligned}
\dot{x}_{i} & =v_{i} \quad i \in\{1, \ldots, N\} \\
\dot{v}_{i} & =\bar{a}\left(v_{i+1}-v_{i}\right)+\bar{b}\left[\bar{k} \cdot\left(x_{i+1}-x_{i}\right)-c-v_{i}\right]
\end{aligned}
$$


Table 1: Variables used in the simulations of Fig. 2.

\begin{tabular}{|c||c||c||c|}
\hline$l_{v}$ & $v_{\max }$ & $d_{s}$ & $k$ \\
\hline $4.5 m$ & $9.75 \mathrm{~m} / \mathrm{s}$ & $6 m$ & 1.2163 \\
\hline \hline$N$ & $l_{r}$ & $a$ & $b$ \\
\hline 22 & $260 m$ & $1.4-209.5$ & $0.1-3$ \\
\hline
\end{tabular}

which has been linearized around the equilibrium:

$$
\begin{aligned}
& x_{i+1}-x_{i}=h_{e q}=\frac{l_{r}}{N} \\
& \dot{x}_{i}=\dot{x}_{i+1}=v_{e q}=V\left(h_{e q}\right)
\end{aligned}
$$

where $l_{r}$ represents the circumference of the ring. Thus, the linearization coefficients are $\bar{k}, \bar{a}, \bar{b}$ and $c$ are defined as:

$$
\begin{aligned}
& \bar{k}=\frac{\partial V\left(h_{e q}\right)}{\partial h}=v_{\max } \frac{1-\left(\tanh \left(h_{e q}-l_{v}-d_{s}\right)\right)^{2}}{1+\tanh \left(l_{v}+d_{s}\right)} \\
& \bar{a}=\frac{a}{\left(h_{e q}\right)^{2}} \quad \bar{b}=b \quad c=-\bar{k} h_{e q}+V\left(h_{e q}\right)
\end{aligned}
$$

Note that the linearized model (12) broadly appears in literature (cf. [19] and reference therein) for both analysis and synthesis problems. It is common to take the human behavior (12) to be homogeneous, thus resulting in

$$
\Gamma(s)=\frac{v_{i}(s)}{v_{i+1}(s)}=\frac{\bar{a} s+\bar{b} \bar{k}}{s^{2}+(\bar{a}+\bar{b}) s+\bar{b} \bar{k}}
$$

which has been derived by taking $y_{i}=v_{i}$. The interested reader can verify that the same transfer function is obtained from taking $y_{i}=v_{i}-v_{i-1}$ which is another typical way to check string stability $[15,17]$.

The state space formulation for the linearized system (12) on a Ring is defined as

$$
\left[\begin{array}{c}
\dot{\chi}_{1} \\
\vdots \\
\dot{\chi}_{N-1} \\
\dot{\chi}_{N}
\end{array}\right]=\left[\begin{array}{cccc}
A_{1} & A_{2} & & O_{2 \times 2} \\
& \ddots & \ddots & \\
& & A_{1} & A_{2} \\
A_{2} & & & A_{1}
\end{array}\right]\left[\begin{array}{c}
\chi_{1} \\
\vdots \\
\chi_{N-1} \\
\chi_{N}
\end{array}\right]+\left[\begin{array}{c}
0 \\
\vdots \\
0 \\
B_{r}
\end{array}\right] u_{r}
$$

with

$$
A_{1}=\left[\begin{array}{cc}
0 & -1 \\
\bar{b} \bar{k} & -\bar{a}-\bar{b}
\end{array}\right] \quad A_{2}=\left[\begin{array}{ll}
0 & 1 \\
0 & \bar{a}
\end{array}\right] \quad B_{r}=\left[\begin{array}{l}
0 \\
1
\end{array}\right]
$$

and $\chi_{i}=\left[x_{i+1}-x_{i}-h_{e q}, \quad v_{i}-v_{e q}\right]^{T}$.

\subsection{Numerical evaluation of Lyapunov and weak ring stability}

Given the system in (16), it is possible to investigate how Lyapunov stability is affected by different combinations of $a$ and $b$ and by the number of vehicles $N$ in the ring. Equations (10) and (11) have few variables that have to be selected beforehand. Table 1 shows the numerical values of these variables in compliance with the experiment in $[10]$.

\subsubsection{Lyapunov stability}

Lyapunov stability on the ring is checked analyzing the eigenvalues of (16). Note that $A$ in (16) has always a zero eigenvalue due to the ring structure. Such eigenvalues arise from the fact that $x_{N}-x_{1}=\left(x_{2}-x_{1}\right)+\cdots+$ $\left(x_{N}-x_{N-1}\right)$. As shown by Lemma 2 in [17] a (structural) zero-eigenvalue does not compromise the stability of the system and therefore it can be neglected for the purpose of Lyapunov analysis. The results are in Fig. 2. The eigenvalues of the ring change their location by varying $N$, decreasing the stability region (bottom-right corner region in Fig. 2) when increasing $N$. To keep the same equilibrium conditions as in (13) and the same $\bar{k}$, the ring length $l_{r}$ is always increased proportionally with $N$. As illustrated in [20] for $N \rightarrow \infty$ the region of Lyapunov stability coincides with $\|\Gamma\|_{\infty} \leq 1$ which is the condition in Definition 1 to guarantee string stability. 


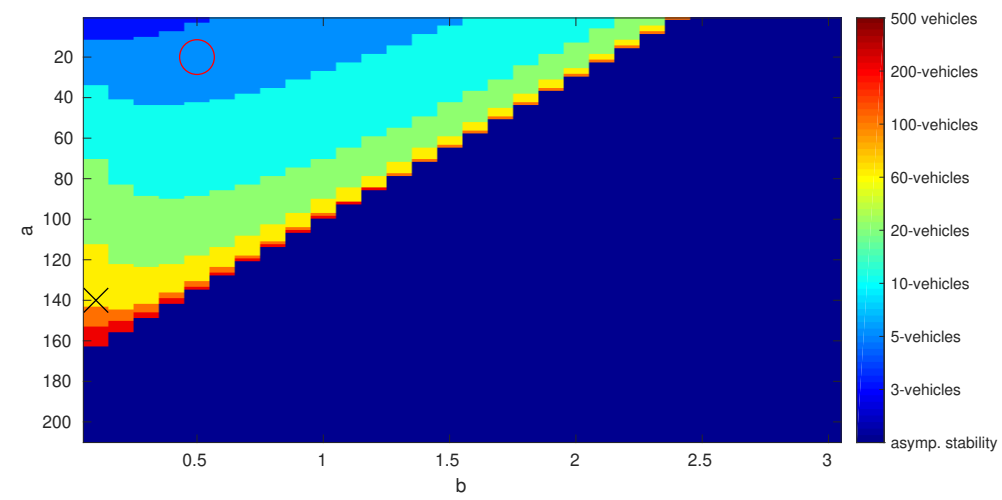

Figure 2: Stability analysis using indirect Lyapunov method. The figure shows, varying $(a, b)$ how many vehicles make the platoon on the ring Lyapunov unstable. Each colour is related to a certain number of vehicles as the colorbar on the right illustrates. The lower-right section is always Lyapunov stable irrespective of the number of vehicles.

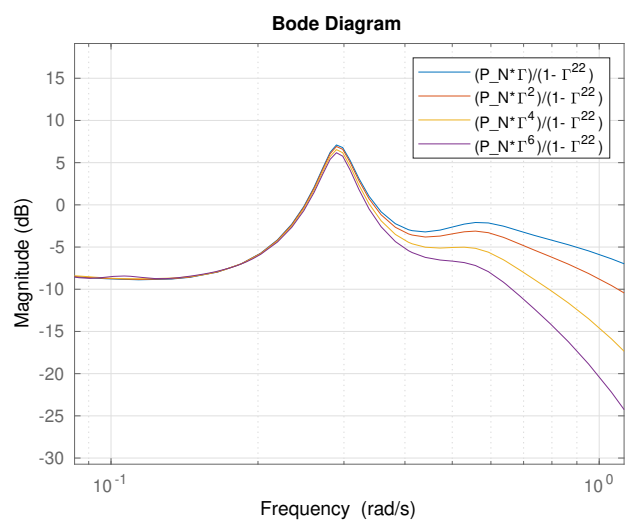

Figure 3: The figure illustrates the Bode plot for $\frac{P_{N} \Gamma^{i}}{1-\Gamma^{N}}$, which is the transfer function between disturbance and vehicle velocity, varying $i \in\{1, \ldots, N\}$. Greater $i$ means moving throughout the platoon with respect to where the disturbance acts. It is possible to see how the peak decreases increasing the value of $i$ and therefore the platoon is weak ring stable.

\subsubsection{Weak ring stability}

Definition 2 gives:

$$
\|\Gamma\|_{\infty} \leq 1 \Rightarrow \text { Lyapunov Stability }
$$

but not viceversa. Therefore, it is possible to find values of $a$ and $b$, that given certain $N$ vehicles, lead to a Lyapunov stable equilibrium even though $\|\Gamma\|_{\infty}>1$. For $N=22$ an example of this situation is given by $a=140, b=0.1$, marked with $\times$ in Fig. 2. Thus, Definition 3 can be used to deal with this case and the results are illustrated in Fig. 3, showing no increase of the peaks of (9).

\section{Mixed platoon}

So far, only human drivers have been considered, consistently with [10]. In this section, considering the experiments in [11], an AV is added to the platoon. We want to determine a design for the AV which is able to stabilise a human-driven unstable platoon, first in Lyapunov and then also in ring stability sense. The AV is the $N$-th vehicle with preceding vehicle 1 . The work [12] proposed a non-linear AV controller

$$
\begin{aligned}
& \dot{x}_{N}=v_{N} \\
& \dot{v}_{N}=K_{\text {veh }}\left(\alpha \cdot v_{\text {target }}+(1-\alpha) v_{1}-v_{N}\right)
\end{aligned}
$$


Table 2: Values used in (24) for Lyapunov analysis

\begin{tabular}{|c||c||c||c||c|}
\hline$a$ & $b$ & $K_{\text {veh }}$ & $\alpha$ & $\delta$ \\
\hline 20 & 0.5 & $10^{-4}-0.02$ & 0.9 & $10-30$ \\
\hline
\end{tabular}

Table 3: Values for simulations with AV: ring unstable case.

\begin{tabular}{|c||c||c||c||c||c|}
\hline$l_{v}$ & $v_{\max }$ & $d_{s}$ & $k$ & $N$ & $l_{r}$ \\
\hline $4.5 \mathrm{~m}$ & $9.75 \mathrm{~m} / \mathrm{s}$ & $6 m$ & 1.2163 & 22 & $260 \mathrm{~m}$ \\
\hline \hline$a$ & $b$ & $K_{\text {veh }}$ & $\alpha$ & $\delta$ & \\
\hline 20 & 0.5 & 0.001 & 0.9 & 23 & \\
\hline
\end{tabular}

where $v_{\text {target }}$ and $\alpha$ are

$$
\begin{aligned}
v_{\text {target }} & =V_{d}+\min \left(\max \left(\frac{x_{1}-x_{N}-7}{\delta}, 0\right), 1\right) \\
V_{d} & =\left(\frac{v_{N}+v_{1}}{2}\right) \Delta x_{s}=\max \left(2 \cdot\left(v_{1}-v_{N}\right), 4\right) \\
\alpha & =\min \left(\max \left(\frac{x_{1}-x_{N}-\Delta x_{s}}{\gamma}, 0\right), 1\right) .
\end{aligned}
$$

At the equilibrium (13), controller (19) can be linearized as

$$
\begin{gathered}
\dot{v}_{N}=K_{v e h}\left((1-\alpha) v_{1}+\alpha\left(\frac{v_{N}+v_{1}}{2}\right)+\right. \\
\left.\alpha \frac{\left(x_{1}-x_{N}-7\right)}{\delta}-v_{N}\right) .
\end{gathered}
$$

and $\dot{x}_{N}=v_{N}$. In a similar way as (15) was obtained, we obtain for the autonomous vehicle:

$$
\Gamma_{A V}(s)=\frac{v_{N}(s)}{v_{1}(s)}=\frac{K_{v e h}\left[\left(1-\frac{\alpha}{2}\right) s+\frac{\alpha}{\delta}\right]}{s^{2}+K_{v e h}\left(1-\frac{\alpha}{2}\right) s+K_{v e h} \frac{\alpha}{\delta}} .
$$

Defining $A_{1}, A_{2}$ and $B_{r}$ as in (17) and

$$
\begin{aligned}
& A_{A V 1}=\left[\begin{array}{cc}
0 & -1 \\
\frac{\alpha K_{v e h}}{\delta} & -\left(K_{v e h}\left(1-\frac{\alpha}{2}\right)+c\right)
\end{array}\right] \\
& A_{A V 2}=\left[\begin{array}{cc}
0 & 1 \\
0 & K_{v e h}\left(1-\frac{\alpha}{2}\right)
\end{array}\right]
\end{aligned}
$$

the state space formulation for the ring with AV becomes:

$$
\left[\begin{array}{c}
\dot{\chi}_{1} \\
\vdots \\
\dot{\chi}_{N-1} \\
\dot{\chi}_{N}
\end{array}\right]=\left[\begin{array}{cccc}
A_{1} & A_{2} & & O \\
& \ddots & \ddots & \\
& & A_{1} & A_{2} \\
A_{A V 2} & \ldots & 0 & A_{A V 1}
\end{array}\right]\left[\begin{array}{c}
\chi_{1} \\
\vdots \\
\chi_{N-1} \\
\chi_{N}
\end{array}\right]+\left[\begin{array}{c}
0 \\
\vdots \\
0 \\
B_{r}
\end{array}\right] u_{r}
$$

\subsubsection{Lyapunov stability with AV}

We illustrate the Lyapunov stability analysis on (24) with numerical values in Tab. 2.

Fig. 4 shows the results of indirect Lyapunov method on (24) for different combinations of $\delta$ and $K_{v e h}$ and for different number of vehicles $N$. The different colors in this case emphasize, for a certain $N$ on the ring, the values of $\delta$ and $K_{v e h}$ which can make the platoon Lyapunov stable. Less vehicles on the ring allow a higher number of $\delta-K_{v e h}$ combinations and also a faster controller provided by higher value of $K_{v e h}$. As appreciable, a proper AV design is able to Lyapunov stabilize a system that would have been unstable with only human drivers for the $a$ and $b$ in Tab. 2.

\subsubsection{Weak ring instability with AV}

Once obtained Lyapunov stability, it is possible to look for ring stability. Since the platoon is heterogeneous and $\|\Gamma\|_{\infty}>1$ for $a$ and $b$ in Tab. 3, marked with $\circ$ in Fig. 2, we are in the weak ring stability case described 


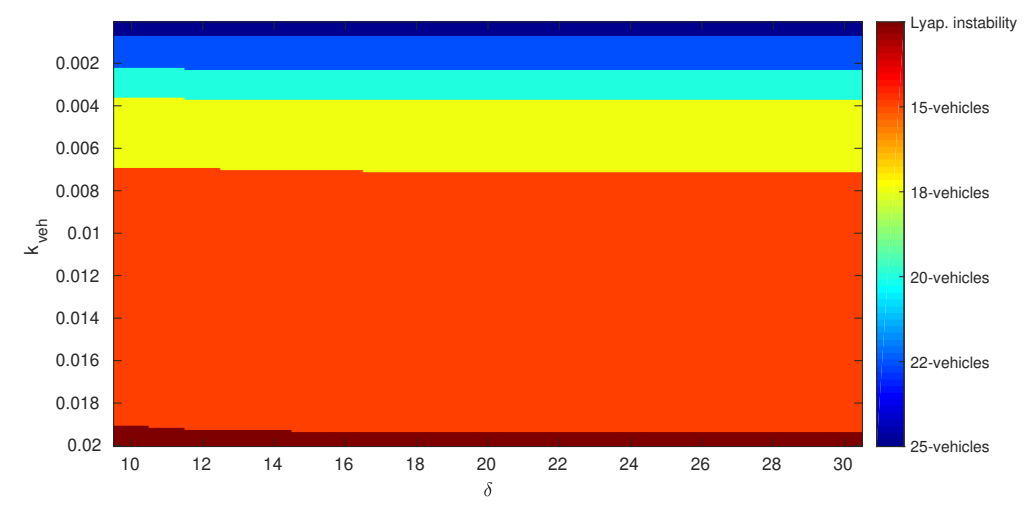

Figure 4: Lyapunov stability for different combinations of $K_{v e h}$ and $\delta$ with fixed $a$ and $b$ given in Tab. 2. The figure shows the $\left(K_{v e h}, \delta\right)$ required to stabilize, in Lyapunov sense, platoons of different $N$. Different colours correspond to different $N$ as the colorbar on the right depicts. For values of $K_{v e h}$ too high the platoons with $N \geq 15$ is Lyapunov unstable.

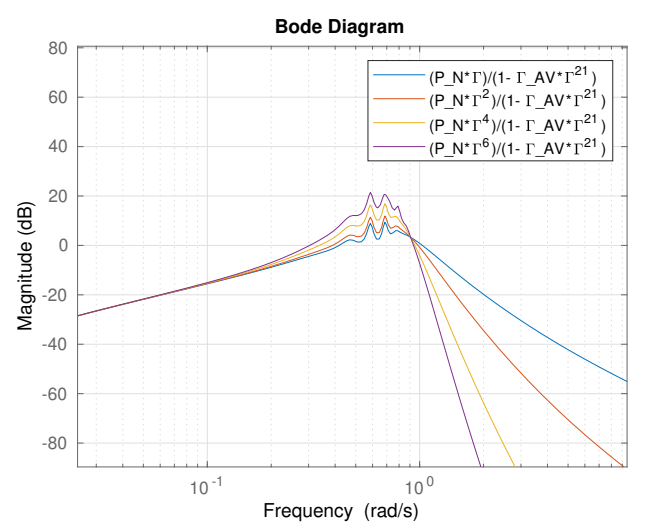

Figure 5: The figure illustrates the transfer function between disturbance and vehicle velocity in a mixed platoon, varying $i \in\{1, \ldots, N\}$. Greater $i$ means moving throughout the platoon with respect to where the disturbance acts. It is possible to see how, increasing the value of $i$, the peak increases. Therefore the platoon is weak ring unstable.

in Definition 3. Lyapunov stability is ensured for the values of $a, b, K_{v e h}$ and $\delta$ in Tab. 3. Hence, fixing $N$ we can study the uniform bound over $i$. The function (8) with only an AV becomes:

$$
F_{i}^{A V(N)}(s)=\frac{\Gamma^{N-i}(s)}{1-\Gamma_{A V}(s) \Gamma^{N-1}(s)} P_{N}(s)
$$

Fig. 5 shows how, starting from the disturbance and going through the vehicles in the platoon, $\left\|F_{i}^{A V(22)}(s)\right\|_{\infty}$ is increasing, denoting an amplification of the disturbance effect. In other words, the AV can achieve Lyapunov stability, but it cannot guarantee ring stability.

\subsubsection{Weak ring stability with AV}

In other situations, such as the one described in Tab. 4, the AV is able to achieve both Lyapunov and weak ring stability. In this case, the selected variables for the human drivers are $a=100$ and $b=0.5$ which, even though make, for $N=22$, the human-drivers platoon Lyapunov unstable (cf. Fig. 2); they describe a behavior which is

Table 4: Numerical values for simulations with AV: ring stable case.

\begin{tabular}{|c||c||c||c||c||c|}
\hline$l_{v}$ & $v_{\max }$ & $d_{s}$ & $k$ & $N$ & $l_{r}$ \\
\hline $4.5 \mathrm{~m}$ & $9.75 \mathrm{~m} / \mathrm{s}$ & $6 m$ & 1.2163 & 22 & $260 \mathrm{~m}$ \\
\hline \hline$a$ & $b$ & $K_{\text {veh }}$ & $\alpha$ & $\delta$ & \\
\hline 100 & 0.5 & 1 & 0.9 & 23 & \\
\hline
\end{tabular}




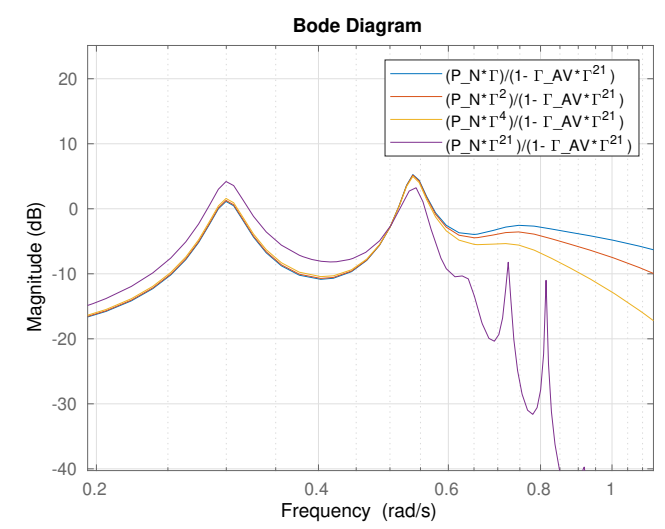

Figure 6: The figure illustrates for values in Tab. 4 the Bode plot for $\frac{P_{N} \Gamma^{i}}{1-\Gamma_{A V} \Gamma^{N-1}}$, which is the transfer function between disturbance and vehicle velocity in a mixed platoon, varying $i \in\{1, \ldots, N\}$. Greater $i$ means moving throughout the platoon with respect to where the disturbance acts. It is possible to see how, increasing the value of $i$, the dominant peak does not increase and therefore the platoon is weak ring stable.

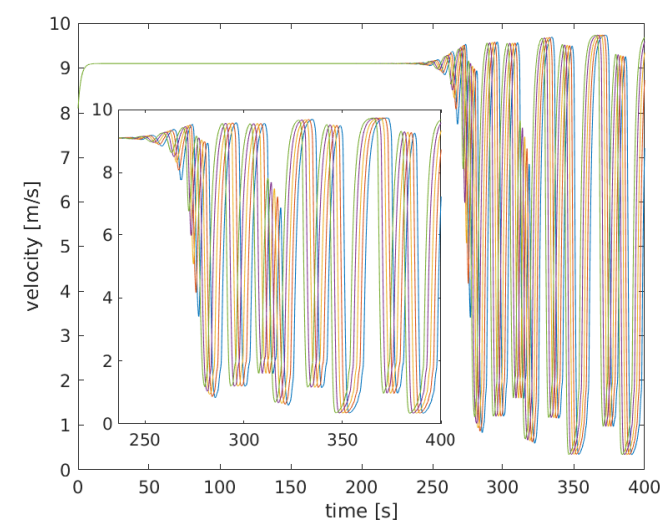

Figure 7: Simulation of the vehicles velocity on the ring with only human drivers (eq. 10) for values in tab. 3. The figure illustrates a typical behavior of a Lyapunov unstable system. The platoon consists in 22 human drivers vehicles of which only 5 are plotted.

easier to stabilize. Consequentially, the $\mathrm{AV}$ controller can afford $K_{v e h}=1$ which provides a faster convergence towards the equilibrium. In frequency domain, this scenario turns into what described in Fig. 6, where the magnitude peaks of the transfer functions, from disturbance to velocities, decrease throughout the platoon.

\section{Simulations of nonlinear platoon dynamics}

This section has the purpose to explore by simulations what is the evolution of the nonlinear platoon dynamics on the ring, with and without the AV: Fig. 7, 8 and 10 illustrate the velocities of certain vehicles in the platoon for all the cases previously analysed. The initial conditions are close to the equilibrium (13).

In Fig. 7 there are only human vehicles with non-linear dynamics (10): only 5 vehicles are plotted, out of 22. Given $a=20$ and $b=0.5$, the equilibrium is Lyapunov unstable and the oscillations in velocity increase over time.

In Fig. 8, replacing a human vehicle with the linear AV controller (20) yields to a Lyapunov stable equilibrium. However, the platoon results ring unstable since moving throughout the platoon the disturbance is amplified (only 8 vehicles out of 22 are plotted). The Bode plot related to this case is in Fig. 5 which shows increasing peaks; this fact can also be verified in time domain by calculating the energy of the error of the vehicles velocities with respect $v_{e q}$

$$
e=\int_{0}^{t_{\text {final }}}\left(v_{i}(t)-v_{e q}\right)^{2} d t
$$

Fig. 9 shows how energy grows throughout the platoon.

Finally, Fig. 10 investigates the case in which the AV can achieve both Lyapunov and weak ring stability 


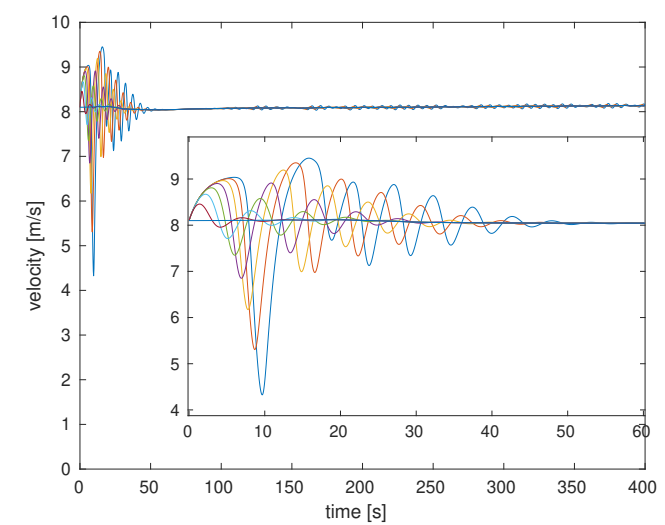

Figure 8: Simulation of the vehicles velocity on the ring with an AV (20) and 21 human drivers (10) for values in tab. 3. The system is Lyapunov stable but ring unstable. Overall, the platoon consists of 22 vehicles of which only the AV and the 7 following human vehicles are plotted.

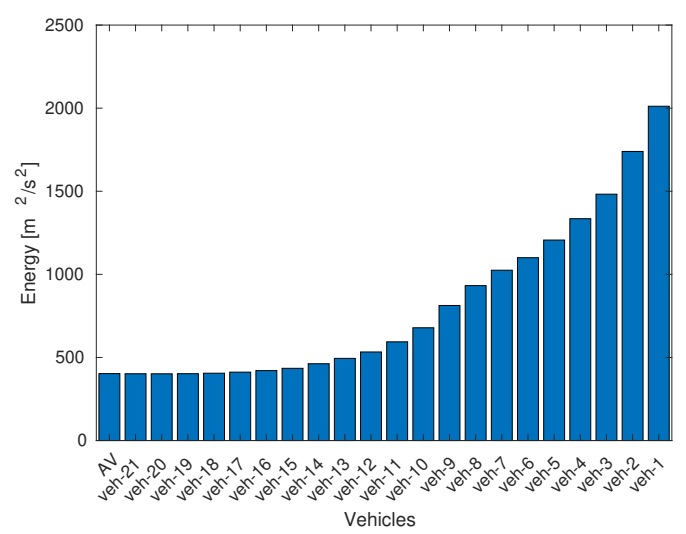

Figure 9: Energy computed as in (26) for the different vehicles in the platoon for Fig. 8 case. The purpose of this figure is mainly to show the energy trend starting from the AV throughout the platoon.

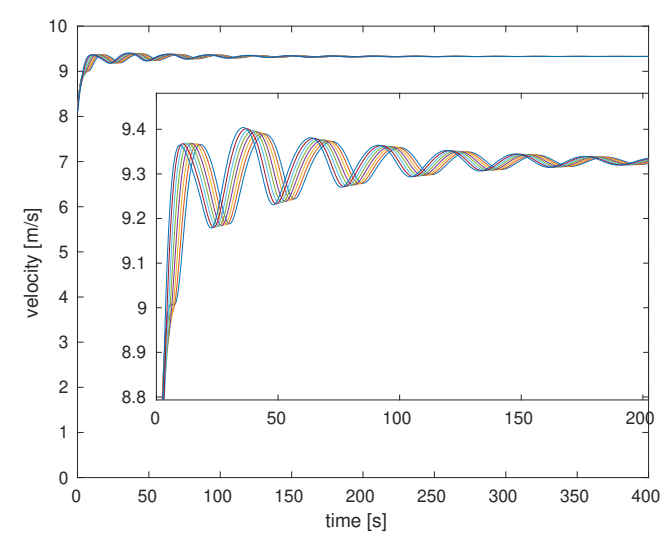

Figure 10: Simulation of the vehicles velocity on the ring with an AV (20) and 21 human drivers (10) for values in tab. 4. The system is Lyapunov and ring stable. Overall, the platoon consists of 22 vehicles of which only the $\mathrm{AV}$ and the 7 following human vehicles are plotted. 


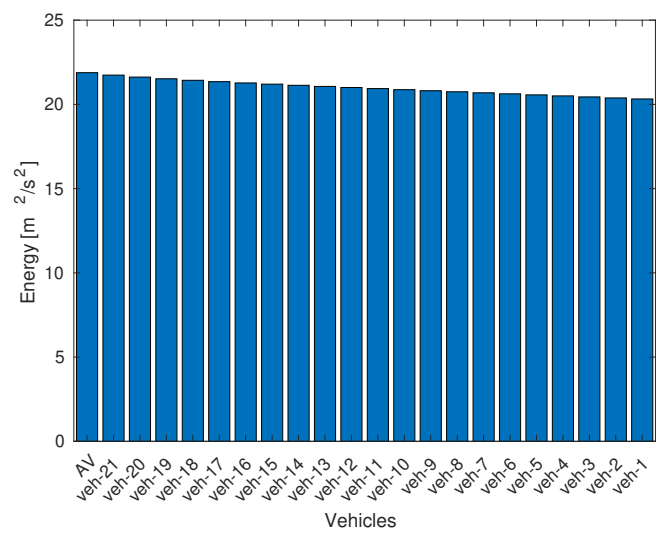

Figure 11: Energy of velocity error computed as in (26) for the different vehicles in the platoon for the case of Fig. 10. The figure shows the energy decrease starting from the AV throughout the platoon.

(Bode plot in Fig. 6): the overshoot amplification typical of string/ring instability is absent, as also confirmed in Fig. 11, reporting the energy in (26).

\section{Conclusion and future works}

The main purpose of this work was to construct a theoretical framework to understand experiments with platoons of vehicles on ring roads, such as $[10,11]$. We have shown that the platoon dynamics can be analyzed by tools available in the linear domain. In view of our definition of weak ring stability, stability of linearized a car-following model on the ring has been discussed. A stabilizing AV was considered and validated in nonlinear simulations.

Future work will include the study of the effects of increasing communication among vehicles, and the study of the relation between ring and Lyapunov instability.

\section{Acknowledgements}

S. Baldi was partly supported by Fundamental Research Funds for the Central Universities grant 4007019109 (RECON-STRUCT) and by special guiding funds for "double first-class" grant 4007019201. M. Lv was partly supported by a "Descartes Fellowship" by the French Embassy in the Netherlands. P. Frasca was partly supported by ANR via project HANDY (ANR-18-CE40-0010). M. L. Delle Monache was supported by the IDEX-IRS 2018 grant "MAVIT - Modeling autonomous vehicles in traffic flow".

\section{References}

[1] L. Davis, "Effect of adaptive cruise control systems on traffic flow," Physical Review E, vol. 69, no. 6, p. 066110, 2004.

[2] F. Knorr, D. Baselt, M. Schreckenberg, and M. Mauve, "Reducing traffic jams via VANETs," IEEE Transactions on Vehicular Technology, vol. 61, no. 8, pp. 3490-3498, 2012.

[3] M. Guériau, R. Billot, N.-E. El Faouzi, J. Monteil, F. Armetta, and S. Hassas, "How to assess the benefits of connected vehicles? a simulation framework for the design of cooperative traffic management strategies," Transportation research part C: emerging technologies, vol. 67, pp. 266-279, 2016.

[4] Y. A. Harfouch, S. Yuan, and S. Baldi, "An adaptive switched control approach to heterogeneous platooning with intervehicle communication losses," IEEE Transactions on Control of Network Systems, vol. 5, no. 3, pp. 1434-1444, 2018.

[5] M. Papageorgiou and A. Kotsialos, "Freeway ramp metering: An overview," IEEE transactions on intelligent transportation systems, vol. 3, no. 4, pp. 271-281, 2002.

[6] L. D. Baskar, B. De Schutter, J. Hellendoorn, and Z. Papp, "Traffic control and intelligent vehicle highway systems: a survey," IET Intelligent Transport Systems, vol. 5, no. 1, pp. 38-52, 2011.

[7] S. Baldi, I. Michailidis, E. B. Kosmatopoulos, A. Papachristodoulou, and P. A. Ioannou, "Convex design control for practical nonlinear systems," IEEE Transactions on Automatic Control, vol. 59, no. 7, pp. 1692-1705, 2014.

[8] V. Milanés, S. E. Shladover, J. Spring, C. Nowakowski, H. Kawazoe, and M. Nakamura, "Cooperative adaptive cruise control in real traffic situations." IEEE Trans. Intelligent Transportation Systems, vol. 15, no. 1, pp. 296-305, 2014.

[9] A. Talebpour and H. S. Mahmassani, "Influence of connected and autonomous vehicles on traffic flow stability and throughput," Transportation Research Part C: Emerging Technologies, vol. 71, pp. 143-163, 2016.

[10] Y. Sugiyama, M. Fukui, M. Kikuchi, K. Hasebe, A. Nakayama, K. Nishinari, S.-i. Tadaki, and S. Yukawa, "Traffic jams without bottlenecks experimental evidence for the physical mechanism of the formation of a jam," New journal of physics, vol. 10, no. 3, p. 033001, 2008. 
[11] R. E. Stern, S. Cui, M. L. Delle Monache, R. Bhadani, M. Bunting, M. Churchill, N. Hamilton, H. Pohlmann, F. Wu, B. Piccoli et al., "Dissipation of stop-and-go waves via control of autonomous vehicles: Field experiments," Transportation Research Part C: Emerging Technologies, vol. 89, pp. 205-221, 2018.

[12] M. L. Delle Monache, T. Liard, A. Rat, R. Stern, R. Badhani, B. Seibold, J. Sprinkle, D. B. Work, and B. Piccoli, "Feedback control algorithms for the dissipation of traffic waves with autonomous vehicles," in Computational Intelligence and Optimization Methods for Control Engineering. Springer, 2019, to appear.

[13] M. Bando, K. Hasebe, A. Nakayama, A. Shibata, and Y. Sugiyama, "Dynamical model of traffic congestion and numerical simulation," Physical review E, vol. 51, no. 2, p. 1035, 1995.

[14] D. C. Gazis, R. Herman, and R. W. Rothery, "Nonlinear follow-the-leader models of traffic flow," Operations research, vol. 9, no. 4, pp. 545-567, 1961.

[15] J. Ploeg, N. Van De Wouw, and H. Nijmeijer, "Lp string stability of cascaded systems: Application to vehicle platooning," IEEE Transactions on Control Systems Technology, vol. 22, no. 2, pp. 786-793, 2014.

[16] D. Swaroop and J. K. Hedrick, "String stability of interconnected systems," IEEE transactions on automatic control, vol. 41, no. 3, pp. 349-357, 1996

[17] J. A. Rogge and D. Aeyels, "Vehicle platoons through ring coupling," IEEE Transactions on Automatic Control, vol. 53, no. 6, pp. 1370-1377, 2008

[18] A. A. Peters, R. H. Middleton, and O. Mason, "Cyclic interconnection for formation control of 1-D vehicle strings," European Journal of Control, vol. 27, pp. 36-44, 2016.

[19] J. Ge, "Connected cruise control design in mixed traffic flow consisting of human-driven and automated vehicles," Ph.D. dissertation, University of Michigan, 2017.

[20] Y. Zheng, J. Wang, and K. Li, "Smoothing traffic flow via control of autonomous vehicles," arXiv preprint arXiv:1812.09544, 2018 